\title{
On the Inhomogeneous Hall's Ray of Period-One Quadratics
}

\author{
Christopher G. Pinner and Dan Wolczuk
}

\section{CONTENTS}

1. Introduction and Statement of Results

2. The Existence of Hall's Ray and Its Asymptotics

3. The Existence of the Gaps

References
This work was performed while Pinner was at the University of Northern British Columbia, Prince George, BC.
For quadratics with period-one negative continued fraction expansions,

$$
\theta=\frac{1}{a-\frac{1}{a-\frac{1}{a-\cdots}}},
$$

we show that the inhomogeneous Lagrange spectrum,

$$
\boldsymbol{L}(\theta):=\left\{\lim \inf _{|n| \rightarrow \infty}|n|\|n \theta-\gamma\|: \gamma \in \mathbb{R}, \gamma \notin \mathbb{Z}+\theta \mathbb{Z}\right\},
$$

contains an inhomogeneous Hall's ray $[0, \mathrm{c}(\theta)]$ with

$$
\mathrm{c}(\theta)=\frac{1}{4}\left(1-\mathrm{O}\left(\mathrm{a}^{-1 / 2}\right)\right) \text {. }
$$

We describe gaps in the spectrum showing that this is essentially best possible. Pictures of computed spectra are included. Investigating such pictures led us to these results.

\section{INTRODUCTION AND STATEMENT OF RESULTS}

For a fixed irrational real number $\theta$ and real $\gamma$ one defines the inhomogeneous approximation constant

$$
M(\theta, \gamma):=\liminf _{|n| \rightarrow \infty}|n||| n \theta-\gamma|| .
$$

By varying $\gamma$ (not of the form $n+m \theta$ ) one obtains the inhomogeneous Lagrange spectrum of $\theta$

$$
\boldsymbol{L}(\theta):=\{M(\theta, \gamma): \gamma \in \mathbb{R}, \gamma \notin \mathbb{Z}+\theta \mathbb{Z}\} \subseteq\left[0, \frac{1}{4}\right] .
$$

Arguably, the inhomogeneous analogue of the classical Lagrange spectrum $\boldsymbol{L}=\left\{M(\theta, 0)^{-1}: \theta \in \mathbb{R}\right\}$ should be the set of $M(\theta, \gamma)^{-1}$ rather than the set of $M(\theta, \gamma)$, but in order to work with more easily illustrated bounded intervals we decided to avoid the unnecessary complication of taking reciprocals (it is of course trivial to translate our results should the reader prefer the convention of inverting everything). We are interested here in the largest interval $[0, c(\theta)]$ contained in this spectrum,

$$
c(\theta):=\sup \{c:[0, c] \subseteq \boldsymbol{L}(\theta)\},
$$


usually referred to as the inhomogeneous Hall's ray. Using a Hall style Cantor dissection argument, $\mathrm{Cu}-$ sick, Moran and Pollington [Cusick et al. 1996] have shown that the larger spectrum of one-sided inhomogeneous constants, $\liminf \operatorname{in}_{n \rightarrow \infty} n\|n \theta-\gamma\|$, contains a non-degenerate Hall's ray when $\theta$ is the golden ratio. A similar argument can in fact be used to show that $c(\theta)>0$ for any quadratic $\theta$ [Crisp et al. $\geq 2001$ ]. In the special case that the partial quotients of $\theta$ tend to infinity the spectrum will consist solely of the ray $\left[0, \frac{1}{4}\right]$ - a result implicit in [Fukasawa 1926] and [Barnes 1956].

Here we examine the spectrum only in the simplest case, when $\theta$ has a period-one negative continued fraction expansion. We use a constructive approach to obtain very precise results (seemingly not obtainable using the Cantor dissection method). Since changing a finite number of partial quotients does not affect the spectrum we shall work with the purely periodic representative, and suppose from now on that

$$
\theta=\frac{1}{2}\left(a-\sqrt{a^{2}-4}\right)=\frac{1}{a-\frac{1}{a-\frac{1}{a-\cdots}}} .
$$

Theorem 1. For $\theta$ of the form (1-2) the spectrum $(1-1)$ contains the interval $[0, c(\theta)]$ with

$$
c(\theta)=\frac{1}{4}\left(1-O\left(\frac{1}{\sqrt{a}}\right)\right) .
$$

More precisely, as $a \rightarrow \infty$,

$$
\begin{aligned}
\frac{1}{4}\left(1-(1+o(1)) \frac{10}{\sqrt{a}}\right) & \\
\leq c(\theta) & \leq \frac{1}{4}\left(1-(1+o(1)) \frac{2 \sqrt{2}}{\sqrt{a}}\right) .
\end{aligned}
$$

Pinner [2000b] has shown how to use an appropriate $\theta$-expansion of $\gamma$ to evaluate $M(\theta, \gamma)$, which in the period-one case reduces to the classical $\beta$-expansion of Rényi [1957] and Parry [1960] (with $\beta=1 / \theta$ ),

$$
\gamma=\sum_{i=1}^{\infty} \frac{1}{2}\left(a-2+t_{i}\right) \theta^{i}
$$

The $t_{i}$ will be a sequence of integers in $[-(a-2), a]$ with the same parity as $a$ (and no blocks $a, a-2, \ldots$, $a-2, a$ or $a, a-2, a-2, \ldots)$. If the sequence does not contain infinitely many endpoint configurations $t_{i}=a$, we have

$$
M(\theta, \gamma)=\frac{1}{4} \frac{M^{*}(\theta, \gamma)}{1-\theta^{2}}
$$

with

$$
\begin{aligned}
M^{*}(\theta, \gamma) & :=\liminf _{i \rightarrow \infty} s^{*}(i) \\
s^{*}(i) & :=\min \left\{s_{1}^{*}(i), s_{2}^{*}(i), s_{3}^{*}(i), s_{4}^{*}(i)\right\}
\end{aligned}
$$

where

$$
\begin{aligned}
& s_{1}^{*}(i):=\left(1-\theta+d_{i}^{-}\right)\left(1-\theta+d_{i}^{+}\right) \\
& s_{2}^{*}(i):=\left(1+\theta+d_{i}^{-}\right)\left(1+\theta-d_{i}^{+}\right), \\
& s_{3}^{*}(i):=\left(1-\theta-d_{i}^{-}\right)\left(1-\theta-d_{i}^{+}\right) \\
& s_{4}^{*}(i):=\left(1+\theta-d_{i}^{-}\right)\left(1+\theta+d_{i}^{+}\right),
\end{aligned}
$$

with

$$
d_{i}^{-}=\sum_{j=1}^{i} t_{i+1-j} \theta^{j}, \quad d_{i}^{+}=\sum_{j=1}^{\infty} t_{i+j} \theta^{j} .
$$

When $\gamma$ does contain infinitely many $t_{i}=a$ one needs to check the minimum of $s_{1}^{*}(i)$ and $s_{2}^{*}(i)$ for both $\gamma$ and its negative $1-\theta-\gamma$ (in this case we have $M^{*}(\theta, \gamma) \leq \theta$, and for large $a$ the value will be small and lie well within the inhomogeneous Hall's ray). When $\gamma$ does not contain any $t_{i}=a$ the expansion for $1-\theta-\gamma$ simply replaces the $t_{i}$ by $-t_{i}$, interchanging $s_{3}^{*}(i), s_{4}^{*}(i)$ and $s_{1}^{*}(i), s_{2}^{*}(i)$. Of course if the sequence $t_{i}$ is eventually periodic with period $r$ then we can replace the liminf by a min over cutting the purely periodic sequence (more precisely a doubly infinite sequence with that period) at the $r$ places in its period. Since the $M(\theta, \gamma)$ obtained from the $\gamma$ with periodic expansions (the $\gamma \in \mathbb{Q}(\theta)$ ) are dense in $\boldsymbol{L}(\theta)$ one would expect computing values for small periods to give a reasonable approximation to the spectrum. The figures on pages 490-491 show the spectra obtained by computing the approximation constants corresponding to all possible $\theta$-expansion periods of length at most 7 . An interface to produce similar pictures can be found at http://ctl.unbc.ca/ CMS/LSC/. In [2000b] the spectrum $\boldsymbol{L}(\theta)$ was described down to the first limit point $\delta_{\infty}$, showing an infinite sequence from the largest point $\delta_{0}=$ $\frac{1}{4}\left(1-(1+o(1)) \frac{2}{a}\right)$ to $\delta_{\infty}=\frac{1}{4}\left(1-(1+o(1)) \frac{4}{a}\right)$. The figures clearly suggest holes beyond the first limit point. Examining the configurations that seemed to correspond to the edges of these holes enabled us to identify $O(\sqrt{a})$ of these gaps extending down to 
$\frac{1}{4}(1-(1+o(1)) \sqrt{8} / \sqrt{a})$, giving the upper bound in Theorem 1.

With

$$
u:= \begin{cases}0 & \text { if } a \text { is even } \\ 1 & \text { if } a \text { is odd }\end{cases}
$$

define

$$
\begin{aligned}
& I_{1}(t):=\left(1-\theta-\frac{\left((2 t-u) \theta+u \theta^{2}\right)}{1-\theta^{2}}\right) \\
& \times\left(1-\theta-\frac{\left(u \theta+(2 t-u) \theta^{2}\right)}{1-\theta^{2}}\right) \\
& I_{2}(t):=\left(1-(2 t-u+3) \theta+\frac{(2+u) \theta^{2}+(2 t-u-2) \theta^{3}}{1-\theta^{2}}\right) \\
& \times\left(1+(1-u) \theta+\frac{(2 t+u-2) \theta^{2}+(2-u) \theta^{3}}{1-\theta^{2}}\right), \\
& E_{1}(t):=\left(1-\theta+\frac{-(2 t-u) \theta+(2-u) \theta^{2}}{1-\theta^{2}}\right) \\
& \times\left(1-\theta+\frac{(2-u) \theta-(2 t-u) \theta^{2}}{1-\theta^{2}}\right)
\end{aligned}
$$

moreover for $t \equiv a(\bmod 2)$ set

$$
E_{2}(t):=\left(1-\theta-t \theta+\frac{(t+2) \theta^{2}+(t-4) \theta^{3}}{1-\theta^{2}}\right)^{2}
$$

and for $t \not \equiv a(\bmod 2)$

$$
\begin{aligned}
E_{2}(t):= & \left(1-(t+1) \theta+(t+2) \theta^{2}+(t-3) \theta^{3}\right)^{2} \\
& -\theta^{2}\left(1+\theta-2 \theta^{2}+\frac{\left((t+3) \theta^{3}+(t-5) \theta^{4}\right)}{1-\theta^{2}}\right)^{2} .
\end{aligned}
$$

Theorem 2. Suppose that $a \geq 10$. With $I_{1}, I_{2}, E_{1}, E_{2}$ as above, there are always the following gaps in the spectrum $\boldsymbol{L}(\theta)$ :

1. $I(t):=\frac{1}{4}\left(1-\theta^{2}\right)^{-1}\left(I_{2}(t), I_{1}(t)\right)$

for $t \in \begin{cases}{[0, \sqrt{2 a-8}-3]} & \text { if } a \text { is even, } \\ {\left[\frac{1}{4}(\sqrt{4 a-3}+1), \sqrt{2 a-8}-2\right]} & \text { if } a \text { is odd. }\end{cases}$

2. $E(t):=\frac{1}{4}\left(1-\theta^{2}\right)^{-1}\left(E_{2}(t), E_{1}(t)\right)$,

$$
\text { for }(2+u) \leq t \leq \sqrt{2 a-6 u+16}-(4-u) \text {. }
$$

The endpoints of these intervals are achieved with expansions $t_{i}$ consisting of the following blocks, where $(a, b,)^{k}$ denotes $k$ repetitions of the block $a, b$ and where $k_{i} \rightarrow \infty$ :

$$
\begin{aligned}
& I_{1}(t):-(2 t-u),-u, \\
& I_{2}(t):(2+u),-(2 t+2-u),(2-u, 2 t+u-2,)^{k_{i}} \\
& \quad(2-u),-(2 t+2-u),(2+u, 2 t-u-2,)^{k_{i}}
\end{aligned}
$$

$$
\begin{aligned}
& E_{1}(t):-(2 t-u),(2-u), \\
& E_{2}(t):(-t,-t,)(t+2, t-4,)^{k_{i}}(t+2) \\
& \text { if } t \equiv a(\bmod 2), \\
&-(t+1),-(t-1),((t+3),(t-5),)^{k_{2}}(t+3), \\
&-(t-1),-(t+1),(t+1),(t-1), \\
&(-(t+3),-(t-5),)^{k_{i}}-(t+3),(t-1),(t+1) \\
& \text { if } t \neq \equiv a(\bmod 2) .
\end{aligned}
$$

Finally it seems reasonable to ask two questions:

Question 1. Can (1-3) be improved to a precise asymptotic result? For example perhaps

$$
c(\theta)=\frac{1}{4}(1-(1+o(1)) \sqrt{8 / a}) .
$$

Indeed can the top of the ray be determined exactly?

Question 2. Is there an absolute constant $c_{0}>0$ such that the spectrum of an arbitrary quadratic $\theta$ always contains a ray $[0, c(\theta)]$ with $c(\theta)>c_{0}$ ? Is it true that $c(\theta) \rightarrow \frac{1}{4}$ as the size of the smallest partial quotient in the period of the continued fraction expansion of $\theta$ tends to infinity? We can claim no numerical evidence to suggest that these are true (or even give an upper bound on the optimal $c_{0}$ ) but it is worth remarking that both do hold for the largest point in the spectrum; see [Pinner 2000a], for example.

\section{THE EXISTENCE OF HALL'S RAY AND ITS ASYMPTOTICS}

We now prove Theorem 1 (assuming Theorem 2, which is proved in Section 3). The upper bound in (1-4) follows from Theorem 2 . The lower bound will follow from Lemma 2 below. The proof is constructive. Since we are only interested in an asymptotic result we shall assume that $a$ is large (one can show the existence of a ray for much smaller values).

Lemma 1. Suppose that $a \geq 4^{8}$ and that

$$
\xi=\left(1-\theta-\left(m_{1}+u_{0}\right) \theta\right)^{2},
$$

with $u_{0} \in\left[\frac{1}{6}(\sqrt{10}-3), \frac{1}{6}(\sqrt{10}+3)\right]$ and $4 \sqrt{a} \leq m_{1} \leq$ $a-10 a^{1 / 4}$. Then for $i \geq 1$ we can successively write

$$
\begin{aligned}
\xi=\left(1-\theta-\sum_{j=1}^{i}\left(m_{j}\right.\right. & \left.\left.+l_{j}\right) \theta^{j}-u_{i} \theta^{i}\right) \\
& \times\left(1-\theta-\sum_{j=1}^{i}\left(m_{j}-l_{j}\right) \theta^{j}-u_{i} \theta^{i}\right)
\end{aligned}
$$




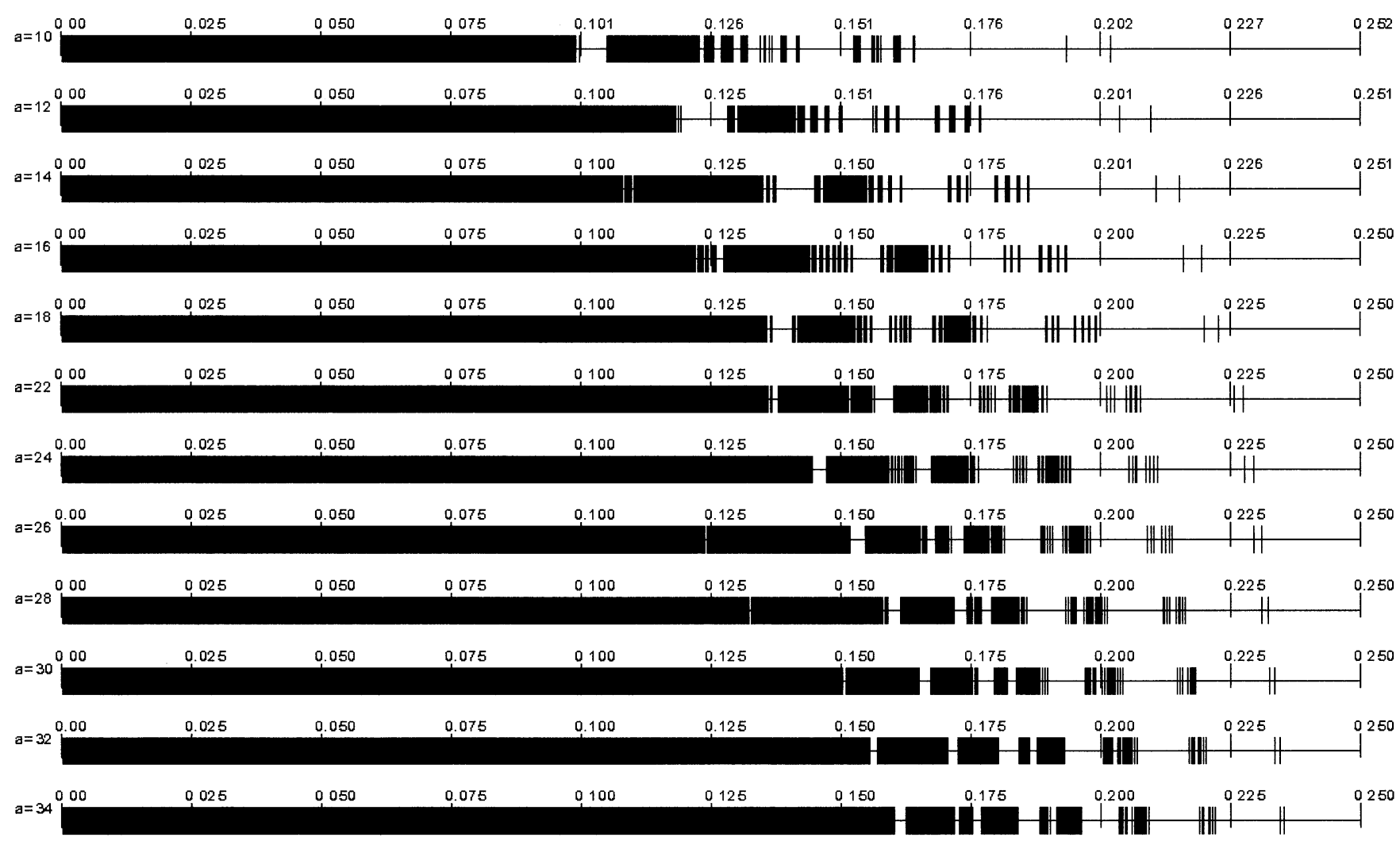

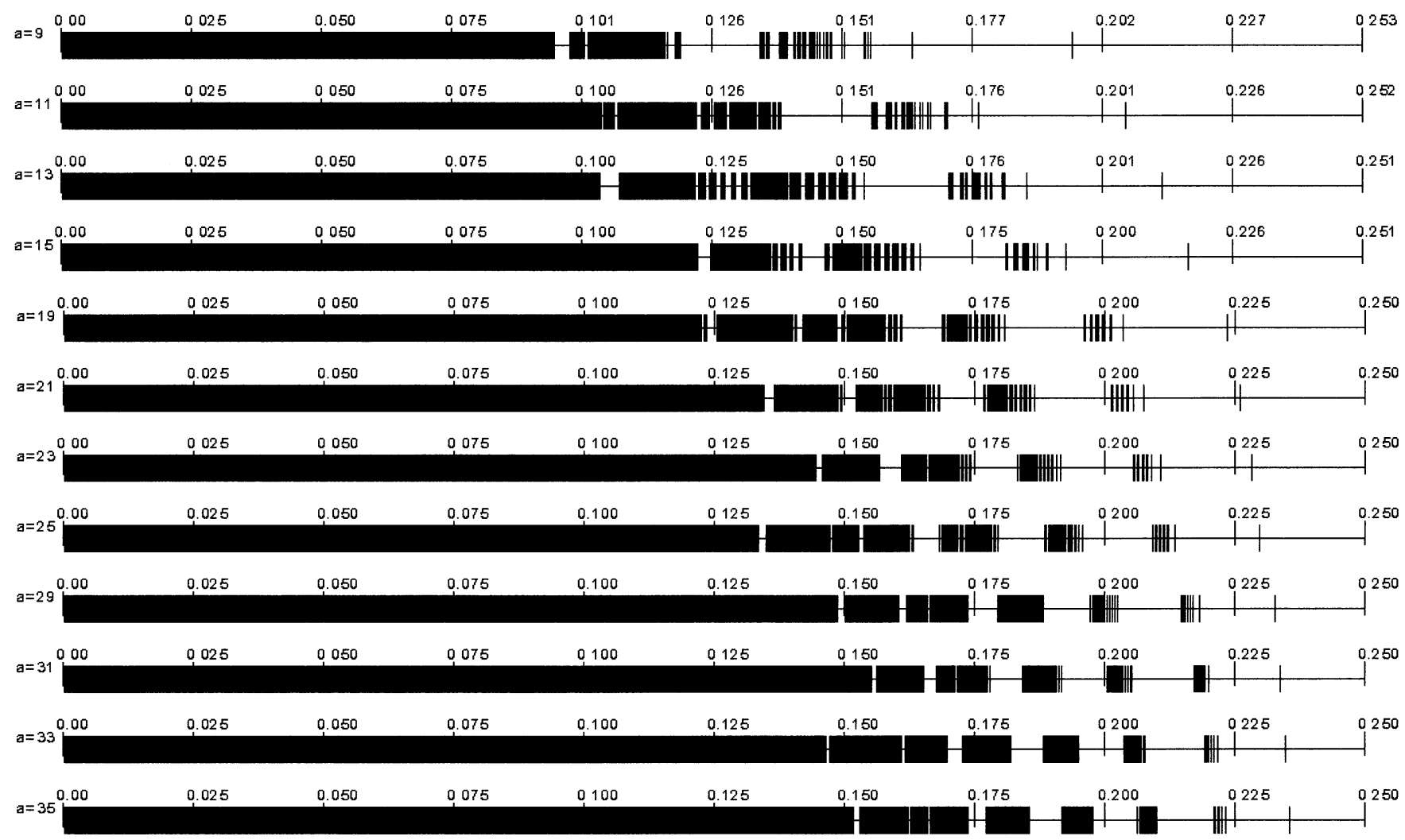

FIGURE 1. Spectra for $\theta$ of period $a$ (values corresponding to $\gamma$ with $\theta$-expansions of period at most seven). 


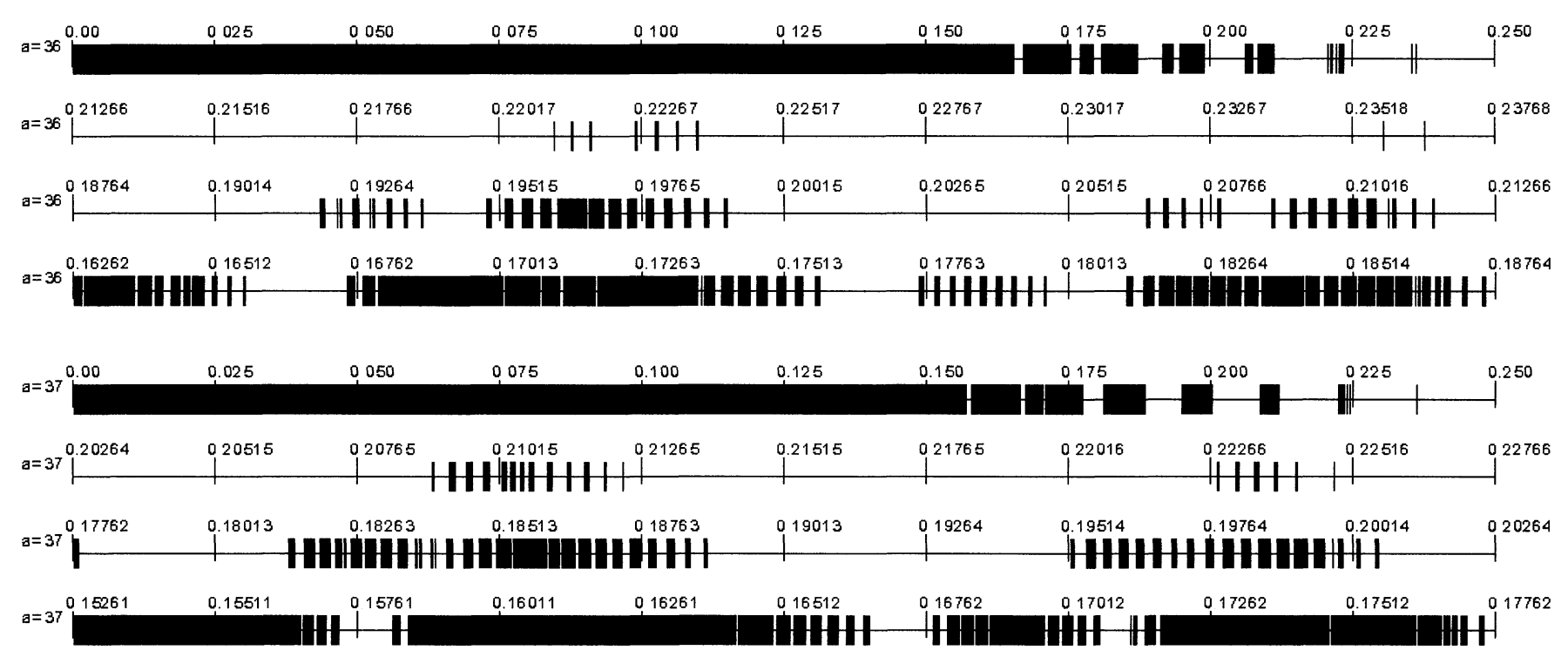

FIGURE 2. Spectra for $a=36,37$ (and $\gamma$ of period at most seven) with zooms.

with integers $m_{i}, l_{i}$, satisfying $m_{i}+l_{i} \equiv a(\bmod 2), \quad$ gives

$\sqrt{2 u_{0}} \sqrt{a-m_{1}}\left(1+29\left(a-m_{1}\right)^{-1 / 2}\right)^{-1}$

$$
\leq l_{1} \leq \sqrt{2 u_{0}} \sqrt{a-m_{1}}
$$

and for $i \geq 2$

$$
\begin{aligned}
0 & \leq u_{i-1} \leq \frac{2 \sqrt{2 u_{0}}}{\sqrt{a-m_{1}}}\left(1+2 a^{-1 / 8}\right) a \theta \\
0 & \leq m_{i}=\left\lfloor u_{i-1} / \theta\right\rfloor \leq \frac{2 \sqrt{2 u_{0}}}{\sqrt{a-m_{1}}}\left(1+2 a^{-1 / 8}\right) a, \\
-1 & \leq l_{i} \leq \frac{\sqrt{a-m_{1}}}{\sqrt{2 u_{0}}}\left(1+\frac{29}{\sqrt{a-m_{1}}}\right) \\
& <\frac{1}{\sqrt{2 u_{0}}} \frac{a}{\sqrt{a-m_{1}}}\left(1+a^{-1 / 8}\right) .
\end{aligned}
$$

Proof. The first step, $i=1$, amounts to solving

$$
l_{1}^{2} \theta=\left(2-2 \theta-2 m_{1} \theta-\left(u_{1}+u_{0}\right) \theta\right)\left(u_{0}-u_{1}\right) .
$$

Take $l_{1}$ to be the integer $l_{1} \equiv m_{1}+a(\bmod 2)$ such that

$$
\sqrt{\frac{\left(2-2 \theta-2 m_{1} \theta-u_{0} \theta\right) u_{0}}{\theta}}=l_{1}+\lambda_{1}
$$

with $0 \leq \lambda_{1}<2$. We have $l_{1}<\sqrt{2\left(a-m_{1}\right) u_{0}}$ and

$$
\begin{aligned}
l_{1} & >\sqrt{\left(2 a-2 \theta-2-2 m_{1}-u_{0}\right) u_{0}}-2 \\
& >\sqrt{2\left(a-m_{1}\right) u_{0}}\left(1+29\left(a-m_{1}\right)^{-1 / 2}\right)^{-1} .
\end{aligned}
$$

Hence

$$
\begin{aligned}
\theta \lambda_{1}^{2}-2 \lambda_{1} \sqrt{\left(2-2 \theta-2 m_{1} \theta-u_{0} \theta\right) u_{0} \theta} & \\
& =-u_{1}\left(2-2 \theta-2 m_{1} \theta-u_{1} \theta\right)
\end{aligned}
$$

$$
\begin{aligned}
0 \leq u_{1} & <\frac{2 \lambda_{1} \sqrt{u_{0} \theta}}{\sqrt{2-2 \theta-2 m_{1} \theta-u_{0} \theta}} \\
& <\frac{4 \sqrt{u_{0}}}{\sqrt{2 a-2 m_{1}-4}}<\frac{4 \sqrt{u_{0}}}{\sqrt{2 a-2 m_{1}}}\left(1+a^{-1 / 4}\right) a \theta .
\end{aligned}
$$

We proceed now by induction on $i$ and, choosing $m_{i+1}=\left\lfloor u_{i} / \theta\right\rfloor$, write

$$
\begin{aligned}
\xi=(1 & \left.-\theta-\sum_{j=1}^{i}\left(m_{j}+l_{j}\right) \theta^{j}-\left(m_{i+1}+\delta_{i+1}\right) \theta^{i+1}\right) \\
& \times\left(1-\theta-\sum_{j=1}^{i}\left(m_{j}-l_{j}\right) \theta^{j}-\left(m_{i+1}+\delta_{i+1}\right) \theta^{i+1}\right)
\end{aligned}
$$

with $0 \leq \delta_{i+1}<1$, and the claim amounts to

$$
\begin{aligned}
\delta_{i+1}(2-2 \theta- & \left.2 \sum_{j=1}^{i+1} m_{j} \theta^{j}-\delta_{i+1} \theta^{i+1}\right) \\
= & l_{i+1}\left(2 \sum_{j=1}^{i} l_{j} \theta^{j}+l_{i+1} \theta^{i+1}\right) \\
& +u_{i+1}\left(2-2 \theta-2 \sum_{j=1}^{i+1} m_{j} \theta^{j}-u_{i+1} \theta^{i+1}\right) .
\end{aligned}
$$

Writing $B:=\sqrt{a-m_{1}}\left(1+29\left(a-m_{1}\right)^{-1 / 2}\right) / \sqrt{2 u_{0}}$ and choosing $l_{i+1} \equiv m_{i+1}+a(\bmod 2)$ so that

$$
\frac{\left(2-2 \theta-2 \sum_{j=1}^{i+1} m_{j} \theta^{j}-\delta_{i+1} \theta^{i+1}\right) \delta_{i+1}}{2 \sum_{j=1}^{i} l_{j} \theta^{j}+B \theta^{i+1}}=l_{i+1}+\lambda_{i+1}
$$


with $0 \leq \lambda_{i+1}<2$, we have

$$
-1 \leq l_{i+1}<\frac{2-2 \theta-2 m_{1} \theta}{2 l_{1} \theta-2 \theta^{2} /(1-\theta)}<\frac{a-m_{1}}{l_{1}} \leq B,
$$

and $u_{i+1}$ is left to satisfy

$$
\begin{aligned}
& u_{i+1}\left(2-2 \theta-2 \sum_{j=1}^{i+1} m_{j} \theta^{j}-u_{i+1} \theta^{i+1}\right) \\
& =\lambda_{i+1}\left(2 \sum_{j=1}^{i} l_{j} \theta^{j}+B \theta^{i+1}\right)+l_{i+1}\left(B-l_{i+1}\right) \theta^{i+1}
\end{aligned}
$$

and, writing $D:=2 \sqrt{2 u_{0}}\left(1+2 a^{-1 / 8}\right) a / \sqrt{a-m_{1}}$, for $a \geq 4^{8}$ we have

$$
\begin{aligned}
0 \leq u_{i+1}< & \frac{\lambda_{i+1}\left(2 l_{1} \theta+2 B \theta^{2} /(1-\theta)\right)+\frac{1}{4} B^{2} \theta^{i+1}}{2-2 \theta-2 m_{1} \theta-2 D \theta^{2} /(1-\theta)} \\
< & \frac{4 \sqrt{u_{0}} \sqrt{2 a-2 m_{1}}}{2 a-2 m_{1}-3} \\
& \times\left(1+\frac{1+10 a^{-1 / 8}}{2 u_{0}} \frac{\theta}{1-\theta}+\frac{11}{32 \sqrt{2} u_{0}^{3 / 2} \sqrt{a}}\right) \\
< & D \theta .
\end{aligned}
$$

Lemma 2. For $a \geq 4^{8}$ the spectrum $\boldsymbol{L}(\theta)$ contains the interval

$$
\left[0, \frac{1}{4}\left(1-c_{0}\left(1+3 a^{-1 / 8}\right) a^{-1 / 2}\right)\right]
$$

with

$$
c_{0}:=2 \sqrt{3}(\sqrt{\sqrt{10}-3}+\sqrt{\sqrt{10}+3})=9.994 \cdots .
$$

Proof. Observe first that any $\xi$ in $\left[U_{1}, U_{2}\right]$, where $U_{1}=10^{2} a^{1 / 2} \theta^{2}$ and $U_{2}:=1-c_{0}\left(1+3 a^{-1 / 8}\right) a^{-1 / 2}$, can be written in the form $\xi=\left(1-\theta-\left(m_{1}+u_{0}\right) \theta\right)^{2}$ with $\frac{1}{2} c_{0}\left(1+\frac{5}{2} a^{-1 / 8}\right) \sqrt{a}<m_{1}<a-10 a^{1 / 4}$ and $u_{0} \in$ $\left[\frac{1}{6}(\sqrt{10}-3), \frac{1}{6}(\sqrt{10}+3)\right]$. Hence we can write $\xi=$ $v v^{\prime}$ with

$$
\begin{aligned}
v & =1-\theta-\sum_{i=1}^{\infty} e_{i} \theta^{i}, \quad e_{i}=m_{i}+l_{i}, \\
v^{\prime} & =1-\theta-\sum_{i=1}^{\infty} e_{i}^{\prime} \theta^{i}, \quad e_{i}^{\prime}=m_{i}-l_{i},
\end{aligned}
$$

where the integers $m_{i}, l_{i}$ satisfy the conditions of Lemma 1 . Clearly then by taking a $\gamma_{0}$ whose sequence $t_{i}$ consists of increasingly long blocks of the form $\cdots-e_{3}^{\prime},-e_{2}^{\prime},-e_{1}^{\prime},-e_{1},-e_{2},-e_{3}, \ldots$, we have $s^{*}(i)=s_{1}^{*}(i) \rightarrow \xi$ for the $\left(t_{i}, t_{i+1}\right)=\left(-e_{1}^{\prime},-e_{1}\right)$. We show that $s^{*}(i)>\xi$ for the remaining $\left(t_{i}, t_{i+1}\right) \neq$ $\left(-e_{1}^{\prime},-e_{1}\right)$. It will be enough to show that for $i \neq 1$

$$
m_{i}+l_{i}+1<m_{1}-\left(l_{1}+1\right)-\frac{\left(l_{1}+1\right)^{2} \theta}{1-\theta-\left(m_{1}+l_{1}+1\right) \theta} .
$$

Since $m_{1}+l_{1}<m_{1}+\sqrt{2 u_{0}} \sqrt{a-m_{1}}<a-130$ we certainly have $\left|d_{i}^{-}\right|,\left|d_{i-1}^{+}\right| \leq\left(\left|t_{i}\right|+1\right) \theta$. Hence, under the assumption $\left(t_{i}, t_{i+1}\right) \neq\left(-e_{1}^{\prime},-e_{1}\right)$,

$s^{*}(i) \geq\left(1-\theta-\left(m_{j}+l_{j}+1\right) \theta\right)\left(1-\theta-\left(m_{j+\varepsilon}+l_{j+\varepsilon}+1\right) \theta\right)$ for some $j \neq 1$ and $\varepsilon= \pm 1$, and (2-1) gives

$$
\begin{aligned}
s^{*}(i) & >\left(1-\theta-\left(m_{j}+l_{j}+1\right) \theta\right)\left(1-\theta-\left(m_{1}+l_{1}+1\right) \theta\right) \\
& >\left(1-\theta-m_{1} \theta\right)^{2}>\xi .
\end{aligned}
$$

Now

$$
\begin{aligned}
\frac{\left(l_{1}+1\right) \theta}{1-\theta-\left(m_{1}+l_{1}+1\right) \theta} & <\frac{\sqrt{2 u_{0}} \sqrt{a-m_{1}}+1}{a-m_{1}-2-\theta-\sqrt{2 u_{0}} \sqrt{a-m_{1}}} \\
& <\frac{3}{\sqrt{a-m_{1}}}
\end{aligned}
$$

and $l_{1}\left(1+3\left(a-m_{1}\right)^{-1 / 2}\right)<\sqrt{2 u_{0}} a / \sqrt{a-m_{1}}$, giving

$$
\begin{aligned}
\left(m_{i}+l_{i}+1\right)+ & \left(l_{1}+1\right)+\frac{\left(l_{1}+1\right)^{2} \theta}{\left(1-\theta-\left(m_{1}+l_{1}+1\right) \theta\right)} \\
& <\left(1+2 a^{-1 / 8}\right)\left(3 \sqrt{2 u_{0}}+\frac{1}{\sqrt{2 u_{0}}}\right) \frac{a}{\sqrt{a-m_{1}}} \\
& <\left(1+2 a^{-1 / 8}\right) \frac{1}{2} c_{0} \frac{a}{\sqrt{a-m_{1}}}=: E .
\end{aligned}
$$

Plainly $E<m_{1}$ for $m_{1}=\frac{1}{2} c_{0}\left(1+\frac{5}{2} a^{-1 / 8}\right) \sqrt{a}$ and $m_{1}=a-10 a^{1 / 4}$ (and all the $m_{1}$ in between by concavity). So (2-1) holds and $M^{*}\left(\theta, \gamma_{0}\right)=\xi$.

Likewise for any $k \geq 1$ a $\gamma_{k}$ made from increasingly long blocks of

$\cdots,-e_{3}^{\prime},-e_{2}^{\prime},-\left(e_{1}^{\prime}+2\right), a, \underbrace{a-2, \ldots, a-2}_{k-1 \text { times }}, e_{1}, e_{2}, \ldots$

will have $s_{2}^{*}(i) \rightarrow \theta^{k} \xi$ when $\left(t_{i}, t_{i+1}\right)=\left(-\left(e_{1}^{\prime}+2\right), a\right)$. The negative of this sequence is simply

$$
\cdots, e_{2}^{\prime}, e_{1}^{\prime}, \underbrace{a-2, \ldots, a-2}_{k-1 \text { times }}, a,-\left(e_{1}+2\right),-e_{2},-e_{3}, \ldots
$$

Trivially, since $e_{j}, e_{j}^{\prime} \leq m_{1}-l_{1}-2$ for $j \neq 1$, we have

$s_{1}^{*}(i) \geq\left(1-\theta-\left(m_{1}+l_{1}+3\right) \theta\right)$

$$
\left(1-\theta-\left(m_{1}-l_{1}-1\right) \theta\right)>\theta v^{\prime}>\theta \xi
$$


while for $t_{i+1} \neq a$

$$
\begin{aligned}
s_{2}^{*}(i) \geq\left(1+\theta-\left(m_{1}+l_{1}+2\right) \theta+a \theta^{2}\right) & \\
\left(1+\theta-(a-2) \theta-a \theta^{2}\right) & >v \theta>\theta \xi .
\end{aligned}
$$

Finally when $\left(t_{i}, t_{i+1}\right)=\left(a-2\right.$ or $\left.e_{1}^{\prime}, a\right)$ we have $s_{2}^{*}(i) \geq(1+\theta)\left(1+\theta-a \theta+\left(e_{1}^{\prime}+1\right) \theta\right)>\theta(1+\theta)>\theta \xi$.

Thus $M^{*}\left(\theta, \gamma_{k}\right)=\theta^{k} \xi$.

Hence for any $k \geq 0$ we can construct a $\gamma$ with $M^{*}(\theta, \gamma)$ taking any value in $\left[\theta^{k} U_{1}, \theta^{k} U_{2}\right]$. Since $\theta U_{2}>U_{1}$ we thereby obtain everything in $\left[0, U_{2}\right]$.

\section{EXISTENCE OF THE GAPS}

We now prove the existence of the gaps given by Theorem 2.

Note that

$$
\begin{aligned}
I_{1}(t)-I_{2}(t) & =\frac{2 \theta^{2}(1-\theta)}{\left(1-\theta^{2}\right)^{2}} \kappa^{\prime}, \\
E_{1}(t)-E_{2}(t) & =\frac{\theta^{2}}{\left(1-\theta^{2}\right)^{2}} \kappa
\end{aligned}
$$

with

$$
\begin{gathered}
\kappa^{\prime}=(1-u)\left(3+(2 t-2) \theta-2 \theta^{2}+\theta^{3}\right)+ \\
\theta(1+\theta)\left(4 t^{2}-2 t+1-a\right)+2 \theta^{2}(1+t \theta), \\
\frac{\kappa}{1-\theta}=(2 a+14-6 u)-(t+4-u)^{2} \\
+\theta t^{2}\left(5+8 \theta+4 \theta^{2}\right)+\theta(8-u) \\
+2 \theta^{2}(1+4 \theta)-2 \theta t(u+2+6 \theta(1+\theta))
\end{gathered}
$$

when $t \equiv a(\bmod 2)$, and

$$
\begin{aligned}
\kappa= & (2 a+13-6 u)-(t+4-u)^{2} \\
& +t^{2} \theta\left(6+3 \theta-2 \theta^{2}\left(1-\theta^{2}\right)(3+2 \theta)\right) \\
& +2 \theta t\left(2-2 u+(u-2) \theta-2 \theta^{2}\left(1+\theta^{2}\right)+11 \theta^{3}\left(1-\theta^{2}\right)\right) \\
& +\theta\left(10-2 u+(u-7) \theta-8 \theta^{2}+14 \theta^{3}-32 \theta^{4}+30 \theta^{5}\right)
\end{aligned}
$$

when $t \not \equiv a(\bmod 2)$. So $I_{2}(t)<I_{1}(t)$ for all $t \geq 0$ when $a$ is even and for $(4 t-1) \geq \sqrt{4 a-3}$ when $a$ is odd, and $E_{2}(t)<E_{1}(t)$ for all $t \leq \sqrt{2 a-6 u+16}-$ $(4-u)$.

We suppose that $\gamma$ has $M^{*}(\theta, \gamma)$ in $\left[I_{2}(t), I_{1}(t)\right]$ (Case I) or $\left[E_{2}(t), E_{1}(t)\right]$ (Case II), with $t$ as in the statement of Theorem 2. Then from the rough lower bounds

$$
\begin{aligned}
E_{2}(t) & \geq(1-(t+1) \theta)^{2}, \\
I_{2}(t) & \geq 1-(2 t+3-u) \theta
\end{aligned}
$$

we can assume that all the $\left|t_{i}\right|$ are at most $2 t+2 v+u$ with $v=1$ in Case I and $v=0$ in Case II (it was shown in [Pinner 2000b] that if $t_{i}=a$ infinitely often then $M^{*}(\theta, \gamma) \leq \theta$ and if $\left|t_{i}\right| \geq k$ infinitely often then $M^{*}(\theta, \gamma)<1-k \theta+\theta^{2}$, where a finite number of $t_{i}$ can be changed without altering $\left.M^{*}(\theta, \gamma)\right)$. Now if we have $\left(t_{i}, t_{i+1}\right)=(-b,-c)$ (or $\left.(-(b+2),(c+2))\right)$ with $b+c=2 l+2 t, l \geq 1$ then (since the remaining $\left|t_{j}\right| \leq 2 t+u+2 v$ ) we have $s_{1}^{*}(i)$ (or $s_{2}^{*}(i)$ ) bounded by

$$
\begin{array}{r}
\left(1-\theta-b \theta+\frac{(2 t+u+2 v) \theta^{2}}{1-\theta}\right)\left(1-\theta-c \theta+\frac{(2 t+u+2 v) \theta^{2}}{1-\theta}\right) \\
=\left(1-\theta-(t+l) \theta+\frac{(2 t+u+2 v) \theta^{2}}{1-\theta}\right)^{2}-(t+l-c)^{2} \theta^{2} .
\end{array}
$$

For $l \geq 1$ this is plainly less than $E_{2}(t)$ and for $l \geq 2$ is less than

$$
\begin{aligned}
B(t):= & \left(1-(t+2) \theta+\frac{(t+3) \theta^{2}+(t-1) \theta^{3}}{1-\theta^{2}}\right)^{2} \\
& =I_{2}(t)+\frac{\theta^{2}}{\left(1-\theta^{2}\right)^{2}} \Gamma
\end{aligned}
$$

where $\Gamma$, defined to be

$$
\begin{aligned}
& (1+2 \theta)\left((t+3-u)^{2}-(2 a-8)\right) \\
& -\theta(4 t(5-u)+50-18 u)-\theta^{2}\left(3 t^{2}+2(5-u) t-u-9\right) \\
& -\theta^{3}\left(4 t^{2}-20 t-6(1-u)\right)+\theta^{4}\left(4 t^{2}+2 u\right),
\end{aligned}
$$

is certainly negative for $(t+3-u) \leq \sqrt{2 a-8}$. So we can assume that each $\left|t_{i}+t_{i+1}\right|$ is at most $2 t+2 v$, and each $\left|t_{i}-t_{i+1}\right|$ is at most $2 t+4+2 v$. Thus if we have $\left(t_{i}, t_{i+1}\right)=(-b,-c)($ or $(-(b+2),(c+2)))$ with $b+c=2 t+2 v$, then (since $t_{i-1} \leq 2 t+2 v+2-b+2 \lambda$ with $\lambda=1$ or 0 , and $t_{i-1}=2 t+2 v+2-b+2 \lambda$ implies that $t_{i-2} \leq b-2-2 \lambda$ and so on) $s_{1}^{*}(i)$ (or $\left.s_{2}^{*}(i)\right)$ is bounded by the quantity $S$ defined as

$$
\begin{array}{r}
\left(1-\theta-b \theta+\frac{(2 t+2+2 v+2 \lambda-b) \theta^{2}+(b-2-2 \lambda) \theta^{3}}{1-\theta^{2}}\right) \\
\times\left(1-\theta-c \theta+\frac{(2 t+2+2 v+2 \lambda-c) \theta^{2}+(c-2-2 \lambda) \theta^{3}}{1-\theta^{2}}\right) \\
=\left(1-(t+1+v) \theta+\frac{(t+2+v+2 \lambda) \theta^{2}+(t+v-2-2 \lambda) \theta^{3}}{1-\theta^{2}}\right)^{2} \\
-(b-t-v)^{2} \theta^{2}\left(\frac{1+2 \theta}{1+\theta}\right)^{2} .
\end{array}
$$

Since $S$ is bounded by $B(t)<I_{2}(t)$ when $\lambda=0$ and $v=1$, in Case I we can successively rule out any $\left|t_{i}-t_{i+1}\right|=2 t+6$ and $\left|t_{i}+t_{i+1}\right|=2 t+2$. So 
$\left|t_{i}-t_{i+1}\right| \leq 2 t+4$ and $\left|t_{i}+t_{i+1}\right| \leq 2 t$. Since $S$ is less than

$E_{2}(t)+4 \theta^{2}-(b-t)^{2} \theta^{2}+ \begin{cases}0 & \text { if } t \equiv a(\bmod 2), \\ \theta^{2}(1+\theta)^{2} & \text { if } t \not \equiv a(\bmod 2),\end{cases}$

(and hence less that $E_{2}(t)$ for $b \neq t$ or $t \pm 1$ ) in Case II we can assume that $\left|t_{i}+t_{i+1}\right| \leq 2 t-2$ and $\left|t_{i}-t_{i+1}\right| \leq$ $2 t+2$ except for the blocks $\pm\left\{t_{i}, t_{i+1}\right\}=\{-t,-t\}$, $\{-(t+2),(t+2)\},\{-(t+1),-(t-1)\}$ or $\{-(t+3)$, $(t+1)\}$. Now if $t \equiv a(\bmod 2)$ and $\left(t_{i}, t_{i+1}\right)=$ $(-t,-t)$ then (since $t_{i-1} \leq t+2$ and $t_{i-1}=t+2$ implies $t_{i-2} \leq t-4$ and $t_{i-2}=t-4$ implies $t_{i-3} \leq$ $t+2$, etc., and the same for $\left.t_{i+2}, t_{i+3}, t_{i+4}, \ldots\right)$ we obtain $s_{1}^{*}(i) \leq E_{2}(t)$ (with equality if the $(-t,-t)$ is preceded and succeeded by perpetual blocks $(t+2)$, $(t-4))$. Likewise for $\left(t_{i}, t_{i+1}\right)=(-(t+2),(t+2))$ we obtain $s_{2}^{*}(i) \leq E_{2}(t)$ with equality only if the preceding and succeeding blocks take the form $(t+2),(t-$ 4 ) and $-(t+2),-(t-4)$, but then $s_{4}^{*}(i+1)$ will be smaller so these can be dismissed. Likewise if $t \not \equiv a$ $(\bmod 2)$ and $\left(t_{i}, t_{i+1}\right)=(-(t+1),(t+3))$ then since

$$
\left(1+\theta+d_{i}^{-}\right) \leq\left(1+\theta-(t+1) \theta+\frac{(t+3) \theta^{2}+(t-5) \theta^{3}}{1-\theta^{2}}\right)
$$

we must have $t_{i+2}=-(t+1)$ (else trivially $s_{2}^{*}(i)<$ $\left.E_{2}(t)\right)$. Hence

$$
\begin{aligned}
s_{2}^{*}(i)=(1+ & \left.\theta-(t+1) \theta+\theta d_{i-1}^{-}\right) \\
& \times\left(1+\theta-(t+3) \theta+(t+1) \theta^{2}-\theta^{2} d_{i+2}^{+}\right), \\
s_{4}^{*}(i+1)=(1+ & \left.\theta-(t+1) \theta+\theta d_{i+2}^{+}\right) \\
\times & \left(1+\theta-(t+3) \theta+(t+1) \theta^{2}-\theta^{2} d_{i-1}^{-}\right) .
\end{aligned}
$$

Thus, writing

$$
d=\min \left\{d_{i-1}^{-}, d_{i+2}^{+}\right\} \leq \frac{(t+3) \theta+(t-5) \theta^{2}}{1-\theta^{2}}
$$

we have

$$
\begin{aligned}
\min \left\{s_{2}^{*}(i), s_{4}^{*}(i+1)\right\} \leq & (1+\theta-(t+1) \theta+\theta d) \times \\
& \left(1+\theta-(t+3) \theta+(t+1) \theta^{2}-\theta^{2} d\right) \\
< & E_{2}(t)
\end{aligned}
$$

Similarly if $t_{i}, t_{i+1}=-(t-1),-(t+1)$ then

$$
\begin{aligned}
& s_{1}^{*}(i) \leq\left(1-\theta-(t-1) \theta+\frac{(t+3) \theta^{2}+(t-5) \theta^{3}}{1-\theta^{2}}\right) \\
& \times\left(1-\theta-(t+1) \theta+\theta d_{i+1}^{+}\right) .
\end{aligned}
$$

Hence $t_{i+2}, t_{i+3}=t+1, t-1$ (else trivially $s_{1}^{*}(i) \leq$ $\left.E_{2}(t)\right)$ and

$$
\begin{aligned}
s_{1}^{*}(i)= & \left(1-\theta-(t-1) \theta+\theta d_{i-1}^{-}\right) \\
& \times\left(1-\theta-(t+1) \theta+(t+1) \theta^{2}\right. \\
& \left.+(t-1) \theta^{3}-\theta^{3}\left(-d_{i+3}^{+}\right)\right), \\
s_{3}^{*}(i+2)= & \left(1-\theta-(t-1) \theta+\theta\left(-d_{i+3}^{+}\right)\right) \\
\times & \quad\left(1-\theta-(t+1) \theta+(t+1) \theta^{2}\right. \\
& \left.\quad+(t-1) \theta^{3}-\theta^{3} d_{i-1}^{-}\right),
\end{aligned}
$$

with

$$
d=\min \left\{d_{i-1}^{-},-d_{i+3}^{+}\right\} \leq \frac{(t+3) \theta+(t-5) \theta^{2}}{1-\theta^{2}}
$$

and $\min \left\{s_{1}^{*}(i), s_{3}^{*}(i+2)\right\} \leq E_{2}(t)$ (with equality if the preceding and succeeding $t_{i-1}, t_{i-2}, \ldots$ and $-t_{i+4}$, $-t_{i+5}, \ldots$ consist of repeated blocks $\left.(t+3),(t-5)\right)$.

Hence in Case II we can assume that all the $\mid t_{i}+$ $t_{i+1} \mid \leq 2 t-2$ and $\left|t_{i}-t_{i+1}\right| \leq 2 t+2$ or that $\gamma$ is of the form claimed to achieve $E_{2}(t)$. Now if each $\left|t_{i}-t_{i+1}\right|$ is at most $2 t+2+2 v$ and each $\left|t_{i}+t_{i+1}\right|$ is at most $2 t$ then, writing $K=\min \{2 t+2+2 v-b, 2 t+2 v-u\}$, observe that if $t_{i}=-b, 0 \leq b \leq 2 t+2 v-u$ then

$$
\begin{aligned}
& s_{2}^{*}(i) \geq\left(1+\theta-\frac{\left(b \theta+(2 t-b) \theta^{2}\right)}{1-\theta^{2}}\right) \\
&\left(1+\theta-\frac{\left(K \theta+(2 t-K) \theta^{2}\right)}{1-\theta^{2}}\right) \\
&\left(1+\theta-\frac{\left((2 t-u+2 v) \theta+(u-2 v) \theta^{2}\right)}{1-\theta^{2}}\right) \\
&\left(1+\theta-\frac{\left((2+u) \theta+(2 t-u-2) \theta^{2}\right)}{1-\theta^{2}}\right),
\end{aligned}
$$

the minimum occurring when $b=(2+u)$ or $2 t+2 v-$ $u$, with this greater than $I_{1}(t)$ or $E_{1}(t)$ as $v=1$ or 0 . Similarly for $s_{4}^{*}(i)$. Hence for the $\gamma$ of interest we need only consider $s_{1}^{*}(i)$ and $s_{3}^{*}(i)$.

Now if $t_{i}=-b$ with $u \leq b \leq 2 t-u$ and all the $\left|t_{i}+t_{i+1}\right| \leq 2 t-2+2 v$ then

$$
\begin{aligned}
s_{1}^{*}(i) \geq(1-\theta+ & \left.\frac{-b \theta-(2 t-2+2 v-b) \theta^{2}}{1-\theta^{2}}\right) \\
& \times\left(1-\theta+\frac{-(2 t-2+2 v-b) \theta-b \theta^{2}}{1-\theta^{2}}\right) .
\end{aligned}
$$

Clearly the minimum occurs when

$$
b=2 t-u \quad \text { or } u-2+2 v,
$$

equalling $I_{1}(t)$ for $v=1$ and $E_{1}(t)$ for $v=0$ (with equality for period $(2 t-u),(u-2+2 v))$. Likewise 
for the $\gamma$ claimed to achieve $E_{2}(t)$ we easily have $s_{1}^{*}(i)>E_{1}(t)$ for $\left\{t_{i}, t_{i+1}\right\} \neq\{t+u, t-u\}$. Hence $E_{2}(t), E_{1}(t)$ and $I_{1}(t)$ are attained as claimed with $M^{*}(\theta, \gamma)>E_{1}(t)$ for the $\gamma$ remaining in Case II. In Case I it remains only to check the $t_{i}=-(2 t+2-u)$. If $a$ is even then (since $t_{i \pm 1} \leq 2$ and $t_{i \pm 1}=2 \mathrm{im}$ plies $t_{i \pm 2} \leq 2 t-2$ and $t_{i \pm 2}=2 t-2$ implies $t_{i \pm 3} \leq 2$ etc.) we must have $s_{1}^{*}(i) \leq I_{2}(t)$ (with equality if the $-(2 t+2)$ is contained inside blocks $(2 t-2,2)^{k}$, $-(2 t+2),(2,2 t-2)^{k}$ with $\left.k \rightarrow \infty\right)$. Similarly if $a$ is odd and $t_{i+1}=1$ we have (since $t_{i-1} \leq 3$ and $t_{i-1}=3$ implies $t_{i-4} \leq 2 t-3$ and $t_{i-4}=2 t-3$ implies $t_{i-4} \leq 3$, while $t_{i+2} \leq 2 t-1$ and $t_{i+2}=2 t-1$ implies $t_{i+3} \leq 1$ and so on) $s_{1}^{*}(i) \leq I_{2}(t)$ (with asymptotic equality for blocks $\left.(2 t-1,1)^{k},-(2 t+1),(3,2 t-3)^{k}\right)$. Likewise if $t_{i-1}=1 \mathrm{using} s_{1}^{*}(i-1)$. Hence $I_{2}(t)$ is achieved as claimed. Finally if the $t_{i}=-(2 t+1)$ all have $t_{i-1}=t_{i+1}=3$ then

$$
\begin{aligned}
s_{1}^{*}(i) \geq & \left(1-(2 t+2) \theta+\frac{3 \theta^{2}-(2 t+1) \theta^{3}}{1-\theta^{2}}\right) \\
& \times\left(1+2 \theta+\frac{-(2 t+1) \theta^{2}+3 \theta^{3}}{1-\theta^{2}}\right) \\
= & I_{1}(t)+\frac{2 \theta^{3}(a-4 t)(a-1)}{\left(1-\theta^{2}\right)^{2}}
\end{aligned}
$$

and $M^{*}(\theta, \gamma)>I_{1}(t)$.

\section{REFERENCES}

[Barnes 1956] E. S. Barnes, "On linear inhomogeneous Diophantine approximation", J. London Math. Soc. 31 (1956), 73-79.

[Crisp et al. $\geq 2001]$ D. Crisp, W. Moran, C. Pinner, and A. Pollington, "Inhomogeneous diophantine approximation and Hall's ray". In preparation.

[Cusick et al. 1996] T. W. Cusick, W. Moran, and A. D. Pollington, "Hall's ray in inhomogeneous Diophantine approximation", J. Austral. Math. Soc. Ser. A 60:1 (1996), 42-50.

[Fukasawa 1926] S. Fukasawa, "Über die Grossenordnung des absoluten Beträges von einer linearen inhomogenen Form, II", Japan J. Math. 3 (1926), 91-106.

[Parry 1960] W. Parry, "On the $\beta$-expansions of real numbers", Acta Math. Hungar. 11 (1960), 401-416.

[Pinner 2000a] C. Pinner, "Lower bounds on the two-sided inhomogeneous approximation constant", preprint, 2000. See http://www.math.ksu.edu/ pinner/ Pubs/Asym.ps.

[Pinner 2000b] C. Pinner, "More on inhomogeneous diophantine approximation", preprint, 2000. See http:// www.math.ksu.edu/ pinner/Pubs/FinalNeg.ps. To appear in J. Th. Nombres Bordeaux.

[Rényi 1957] A. Rényi, "Representations for real numbers and their ergodic properties", Acta Math. Acad. Sci. Hungar. 8 (1957), 477-493.

Christopher G. Pinner, Department of Mathematics, 138 Cardwell Hall, Kansas State University, Manhattan, KS 66506 (pinner@math.ksu.edu)

Dan Wolczuk, Mathematics and Computer Science, University of Northern British Columbia, 3333 University Way, Prince George, B.C., Canada V2N 4 Z9 (wolczu0@unbc.ca) 
